\title{
Estudio de los factores pronosticadores de la sobrevida del cáncer diferenciado del tiroides*
}

\author{
Drs. MIGUEL DOMÍNGUEZ E. ${ }^{1}$, JOSÉ TORRES O. ${ }^{2}$, GILBERTO PÉREZ P. ${ }^{1}$, \\ ROBERTO VOLPATO V. ${ }^{2}$, ALFREDO JADRESIC V. ${ }^{1}$, ENRIQUE LÓPEZ C. ${ }^{1}$ \\ 1 Departamento de Medicina Interna Occidente, Facultad de Medicina, Universidad de Chile. \\ Servicio de Salud Metropolitano Occidente, Hospital San Juan de Dios. \\ 2 Departamento de Cirugía Occidente, Facultad de Medicina, Universidad de Chile, Hospital San Juan de Dios. \\ Santiago, Chile.
}

\begin{abstract}
Prognostic factors study of differentiated thyroid cancer

The aim of this investigation was to evaluate the prognostic factors in patients with differentiated thyroid carcinoma treated and followed for a long period of time. Patients and Method: The following prognostic variables: gender, age at diagnosis, goiter characteristics, histology, differentiation grades, tumor anatomical extension, lymph node and distant initial metastasis and post treatment evolution were studied in a historical cohort of thyroid carcinoma. Surgical treatment was subtotal and total thyroidectomy plus lymphatic cervical selective neck dissection. Radioiodine was employed en 59.2\% and thyroid hormone replacement was used indefinitely. Kaplan Meyer analisis was employed to plot survival curves, log Rank test was used to establish the significance of each variable and square chi to determine differences among groups. Cox model was used to identify prognostic variables. Results: A group of 477 patients with differentiated thyroid carcinoma treated at the Hospital San Juan de Dios, from 1948 to 1992, were studied. Median survival rate was $87 \%$ at the end of observation period. Univariate analysis showed significant differences for age, tumor anatomical extension and histological grade on survival, for the entire serie. According to Cox model method, initial distant metastasis, age $>40$ years, anatomical extension, tumor size $>10 \mathrm{~mm}$, were determinant for unfavorable prognosis for the papillary cancer; distant metastasis, anatomical extension and age at the time of diagnosis were determinant for unfavorable prognosis for the follicular cancer. Conclusion: Overall survival was $87 \%$. Distant metastasis at the time of diagnosis: age older than 40 years, anatomical extension and the size of the tumor were determinant of survival prognosis.
\end{abstract}

Key words: Differentiated thyroid cancer, survival rate, prognostic factors.

\section{Resumen}

El objetivo de esta investigación fue estudiar la supervivencia y los factores del pronóstico del cáncer diferenciado del tiroides tratado y seguido por un período de observación prolongado. Pacientes y Método: En una cohorte histórica prospectiva de cáncer de tiroides diagnosticada por biopsia quirúrgica, se analizó las variables:

\footnotetext{
*Recibido el 12 de mayo de 2010 y aceptado para publicación el 28 de marzo de 2011.

Correspondencia: Dr. Miguel Domínguez E. Los Tuliperos Sur 13.094, Santiago, Chile. mdominguez@med.uchile.cl
} 
género, edad en el momento del diagnóstico, semiología del bocio, histología, grado de diferenciación, extensión anatómica del tumor, la presencia de metástasis linfáticas y a distancia iniciales y la evolución post tratamiento como factores pronosticadores de la sobrevida de los pacientes. La cirugía practicada consistió en tiroidectomía sub total y total más disección linfática cervical selectiva. El método de Kaplan y Meier se usó para diseñar curvas de supervivencia, log Rank test para la significación de cada variable y chi cuadrado para definir la diferencias entre los grupos. El método de Cox se utilizó para identificar las variables del pronóstico. Resultados: 477 pacientes con cáncer diferenciado del tiroides tratados en el Hospital San Juan de Dios desde 1948 hasta 1992. El $59,2 \%$ recibió yodo 131 , todos con tratamiento hormonal de frenación indefinidamente. La supervivencia media fue de $87 \%$ al final del período de observación. El análisis univariable demostró diferencias significativas para la edad, extensión anatómica y grado histológico. Determinan un pronóstico desfavorable para el cáncer papilar: las metástasis a distancia iniciales ( $\mathrm{p}<0,0001)$, la edad $>$ de 40 años $(\mathrm{p}<0,0018)$ y la extensión anatómica; en el cáncer folicular fueron las metástasis a distancia, extensión anatómica y la edad en el momento del diagnóstico. Conclusión: La supervivencia fue de 87\%. Las variables metástasis a distancia en el momento del diagnóstico, edad mayor de 40 años, extensión anatómica y el tamaño del tumor fueron determinantes del pronóstico en la supervivencia de los pacientes.

Palabras clave: Cáncer diferenciado de tiroides, supervivencia, factores pronosticadores.

\section{Introducción}

El cáncer del tiroides es la neoplasia maligna más frecuente del sistema endocrino, sin embargo, constituye menos del $1 \%$ de todos los tumores malignos del cuerpo humano ${ }^{1}$. La incidencia anual en varias experiencias extranjeras fluctúa entre 0,5 y 10 casos por 100.000 habitantes; se calcula que en los Estados Unidos de Norteamérica la incidencia anual es de 20.000 casos, con una mortalidad de 1.600 pacientes por $a \tilde{n} o^{2}$.

La conducta biológica de esta enfermedad es en general de baja malignidad, por lo que su evolución es muy lenta, su curso muy prolongado y generalmente asintomático, en la mayoría de los pacientes ${ }^{3}$.

Diversos factores pronosticadores tales como la edad del paciente en el momento del diagnóstico, el tipo histológico, tamaño del tumor, la invasión de los tejidos vecinos y otras variables, han sido motivo de particular estudio por autores extranjeros ${ }^{4-8}$.

El objetivo de esta investigación fue estudiar los factores del pronóstico en la supervivencia del cáncer diferenciado del tiroides tratado y seguido por un período de observación prolongado y evaluar la influencia del género, histología, grado de diferenciación y extensión anatómica del tumor, la presencia de metástasis linfáticas y a distancia iniciales y la evolución post tratamiento en la supervivencia de los pacientes tratados.

\section{Material y Método}

Corresponde a una cohorte histórica prospectiva de cáncer de tiroides diagnosticado por biopsia quirúrgica. Los criterios de inclusión fueron hombres o mujeres con diagnóstico primario de cáncer diferenciado del tiroides operados en el Hospital San Juan de Dios. En la presente serie se incluyó pacientes ingresados desde los años 1948 a 1992. Se excluyeron todos los pacientes con tratamiento previo en otros centros. El estudio preoperatorio inicial, consistió en la historia y examen físico, estudio hormonal, cintigrafía tiroidea y $\mathrm{Rx}$ de tórax. A partir del inicio de la década de los 80, se empleó la ecotomografía cervical y la punción biopsia con aguja fina. La tomografía axial computada se utilizó en casos seleccionados.

Todos los pacientes fueron tratados quirúrgicamente (tiroidectomía total y subtotal) más disección linfática cervical en casos seleccionados con mayor riesgo de metástasis.

El empleo del yodo radioactivo se utilizó sólo en aquellos pacientes con factores de mal pronóstico: mayores de 40 años, tumores de gran tamaño, extratiroideos, invasores, y con metástasis a distancia. Todos los pacientes fueron mantenidos con tratamiento hormonal supresivo permanente.

Se analizaron las variables: género, edad en el momento del diagnóstico, semiología del bocio, tipo histológico, grado de diferenciación histológica, extensión anatómica del tumor, la presencia de metástasis linfáticas y a distancia iniciales y la evolución post tratamiento como factores pronosticadores de la sobrevida de los pacientes.

El seguimiento de los pacientes se efectuó en la Unidad de Endocrinología del Hospital San Juan de Dios y consistió en evaluación clínica y los siguientes exámenes de laboratorio: TSH, T3, T4, Tg, anticuerpos anti-Tg y estudios por imágenes.

Todas las biopsias fueron informadas en Anatomía Patológica. Tanto los carcinomas papilares como los foliculares fueron clasificados siguiendo el criterio de extensión anatómica establecido por Woolner ${ }^{4}$; para los papilares: en microcarcinomas, intratiroideos y extratiroideos y para los foliculares: en mínimamente invasores e invasores. 
El método de Kaplan y Meier se usó para diseñar las curvas de supervivencia, el log Rank test para la significación de cada variable. Para establecer la diferencia entre los promedios de cada tipo de cáncer se utilizó el test Student y el Chi cuadrado, con un error alfa del 5\%. Finalmente se empleó el modelo de Cox (análisis de riesgos proporcionales) para determinar el valor de las variables independientes en la supervivencia $(\alpha<<0,005: s)^{19}$.

\section{Resultados}

Durante el período de estudio fueron tratados 477 pacientes con cáncer diferenciado del tiroides. El 100\% de los pacientes completó el período de seguimiento; con un promedio de 132 meses y una mediana de 120. La edad en el momento del diagnóstico fluctuó entre 4 y 84 años con un promedio de 42,6 años y una mediana de 41. La distribución por género demostró un predominio del sexo femenino (86,8\%); la distribución por grupos etarios demostró una tasa de $50,5 \%$ para los mayores de 40 años. El bocio uninodular se encontró en el 54,5\% y el multinodular en el $45,5 \%$ de los pacientes.

Las características clínicas y anatomopatológicas de los pacientes aparecen en la Tabla 1. Se pudo observar que hubo un predominio del cáncer papilar 69,4\%. En el 65\% el tamaño del tumor fue mayor a $10 \mathrm{~mm}$ y en 303 se pudo determinar la gradación histológica, que demostró un $58 \%$ de los tumores con Grado II a III de Broders.

La extensión anatómica en 331 carcinomas papilares fue: $16,6 \%$ microcarcinomas, $50,4 \%$ intratiroideos y $33 \%$ extratiroideos; en 146 carcinomas foliculares, la forma mínimamente invasora se encontró en el 75,5\% y la invasora en el $24,5 \%$.

Las metástasis linfáticas iniciales fueron detectadas en el $44 \%$ de los carcinomas papilares y en el 9\% de los foliculares en el momento del tratamiento quirúrgico $(\mathrm{p}<0,0001)$ y las distantes en el 6,8\% de los carcinomas papilares y en $4 \%$ de los foliculares.

El análisis univariado de supervivencia demostró diferencias significativas en las distintas variables (Tabla 2): edad en el momento del diagnóstico mayor de 40 años $(\mathrm{p}<0,0003)$ (Figura 1), la extensión anatómica intratiroídeo vs extratiroídeo $(\mathrm{p}<0,0001)$ (Figura 2). Hubo una diferencia significativa en la supervivencia según el grado histológico del tumor, grado I versus grado II-III ( $p<0,003$ ) (Figura 3 ) y entre los pacientes con o sin metástasis iniciales linfáticas y a distancia (Figura 4).

En la variable género $(\mathrm{p}<0,05)$, el tipo histológico, carcinoma papilar versus carcinoma folicular, no hubo diferencias estadísticamente significativas.

La tasa de supervivencia de toda la serie fue de $87 \%$; la de mortalidad al final del período de observación fue $13 \%$. Cuarenta y un pacientes fallecieron por la enfermedad $(8,6 \%)$ y 19 por otra causa $(4,4 \%)$.

El estudio de la supervivencia en toda la serie, utilizando el modelo probabilístico de Cox en los 419 pacientes cuyos datos fueron debidamente consignados, eligió como variables predictoras del pronóstico: las metástasis a distancia $(\mathrm{p}<0,0001)$; la edad $>$ de 40 años $(\mathrm{p}<0,001)$; la extensión anatómica (intratiroideo versus extratiroideo para el cáncer papilar y en el folicular la extensión anatómica mínimamente invasor versus invasor) $(\mathrm{p}<0,01)$.

Tabla 1. Características clínicas y anatomopatológicas de 477 pacientes con cáncer diferenciado del tiroides

\begin{tabular}{|c|c|c|}
\hline $\begin{array}{l}\text { Género } \\
\text { Masculino } \\
\text { Femenino }\end{array}$ & $\begin{array}{r}63 \\
414\end{array}$ & $\begin{array}{l}13,2 \% \\
86,8 \%\end{array}$ \\
\hline $\begin{array}{l}\text { Edad en el momento diagnóstico } \\
\text { < de } 40 \text { años } \\
>\text { de } 40 \text { años }\end{array}$ & $\begin{array}{l}236 \\
241\end{array}$ & $\begin{array}{l}49,5 \% \\
50,5 \%\end{array}$ \\
\hline $\begin{array}{l}\text { Tipo de bocio* } \\
\text { Uninodular } \\
\text { Multinodular }\end{array}$ & $\begin{array}{l}259 \\
218\end{array}$ & $\begin{array}{l}54,5 \% \\
45,5 \%\end{array}$ \\
\hline $\begin{array}{l}\text { Tipo histológico } \\
\text { Papilar } \\
\text { Folicular }\end{array}$ & $\begin{array}{l}331 \\
146\end{array}$ & $\begin{array}{l}69,4 \% \\
30,6 \%\end{array}$ \\
\hline $\begin{array}{l}\text { Tamaño del tumor } \\
\text { < de } 10 \mathrm{~mm} \text { de diámetro } \\
>\text { de } 10 \mathrm{~mm} \text { de diámetro }\end{array}$ & $\begin{array}{l}167 \\
310\end{array}$ & $\begin{array}{l}35 \% \\
65 \%\end{array}$ \\
\hline $\begin{array}{l}\text { Extensión anatómica del cáncer papil } \\
\text { Microcarcinoma } \\
\text { Intratiroideo } \\
\text { Extratiroideo }\end{array}$ & $\begin{array}{r}55 \\
167 \\
109\end{array}$ & $\begin{array}{l}16,6 \% \\
50,4 \% \\
33,0 \%\end{array}$ \\
\hline $\begin{array}{l}\text { Extensión anatómica del cáncer folic } \\
\text { Mínimamente invasor } \\
\text { Invasor }\end{array}$ & $\begin{array}{r}110 \\
36\end{array}$ & $\begin{array}{l}75,5 \% \\
24,5 \%\end{array}$ \\
\hline $\begin{array}{l}\text { Grado histológico* } \\
\text { Grado I de Broders } \\
\text { Grado II a III de Broders }\end{array}$ & $\begin{array}{l}127 \\
176\end{array}$ & $\begin{array}{l}42 \% \\
58 \%\end{array}$ \\
\hline $\begin{array}{l}\text { Metástasis iniciales }(185 p=41 \%) \\
\text { - Linfáticas } \\
\text { - A distancia }\end{array}$ & $\begin{array}{l}\text { Ca. } \\
\text { papilar } \\
44 \% \\
6,8 \%\end{array}$ & $\begin{array}{c}\text { Ca. } \\
\text { folicular } \\
9 \% \\
4 \%\end{array}$ \\
\hline
\end{tabular}

*consignado en 303 pacientes. 
ESTUDIO DE LOS FACTORES PRONOSTICADORES DE LA SOBREVIDA DEL CÁNCER DIFERENCIADO DEL TIROIDES

Tabla 2. Resultados obtenidos de las curvas de supervivencia en 477 pacientes seguidos hasta por 44 años (análisis univariable)

\begin{tabular}{|c|c|c|}
\hline Variable & Valor estadístico & Significación \\
\hline Género ( $\mathrm{F}$ vs $\mathrm{M}$ ) & $\mathrm{p}<0,0562$ & ns \\
\hline Edad $(>40$ años $\mathrm{y}<40$ años $)$ & $\mathrm{p}<0,0003$ & s \\
\hline $\begin{array}{l}\text { Tamaño del tumor* } \\
<\text { de } 10 \mathrm{~mm} \text { vs }>\text { de } 10 \mathrm{~mm}\end{array}$ & $\begin{array}{l}\text { Consignado en un } \mathrm{n}^{\mathrm{o}} \text { insuficiente de pacientes para este } \\
\text { método estadístico. } \\
\mathrm{p}<0,917\end{array}$ & - \\
\hline $\begin{array}{l}\text { Tipo histológico } \\
\text { Ca. papilar vs. ca. folicular }\end{array}$ & $\mathrm{p}<0,2611$ & ns \\
\hline $\begin{array}{l}\text { Extensión anatómica cáncer papilar: } \\
\text { - Intra tiroideo** vs extra tiroideo }\end{array}$ & $\mathrm{p}<0,0001$ & $\mathrm{~s}$ \\
\hline $\begin{array}{l}\text { Extensión anatómica cáncer folicular } \\
\text { - Mínimamente invasor vs invasor }\end{array}$ & $\mathrm{p}<0,0001$ & s \\
\hline $\begin{array}{l}\text { Grado histológico en } 303 \text { pacientes } \\
\text { G }^{\circ} \text { I vs } G^{\circ} \text { II y III }\end{array}$ & $\mathrm{p}<0,0003$ & s \\
\hline Metástasis iniciales & & \\
\hline Linfáticas & $\mathrm{p}<0,0006$ & $\mathrm{~s}$ \\
\hline A distancia & $\mathrm{p}<0,0001$ & $\mathrm{~s}$ \\
\hline
\end{tabular}

*En 58 pacientes no hubo suficiente información que permitiera su análisis. **Incluye los microcarcinomas.
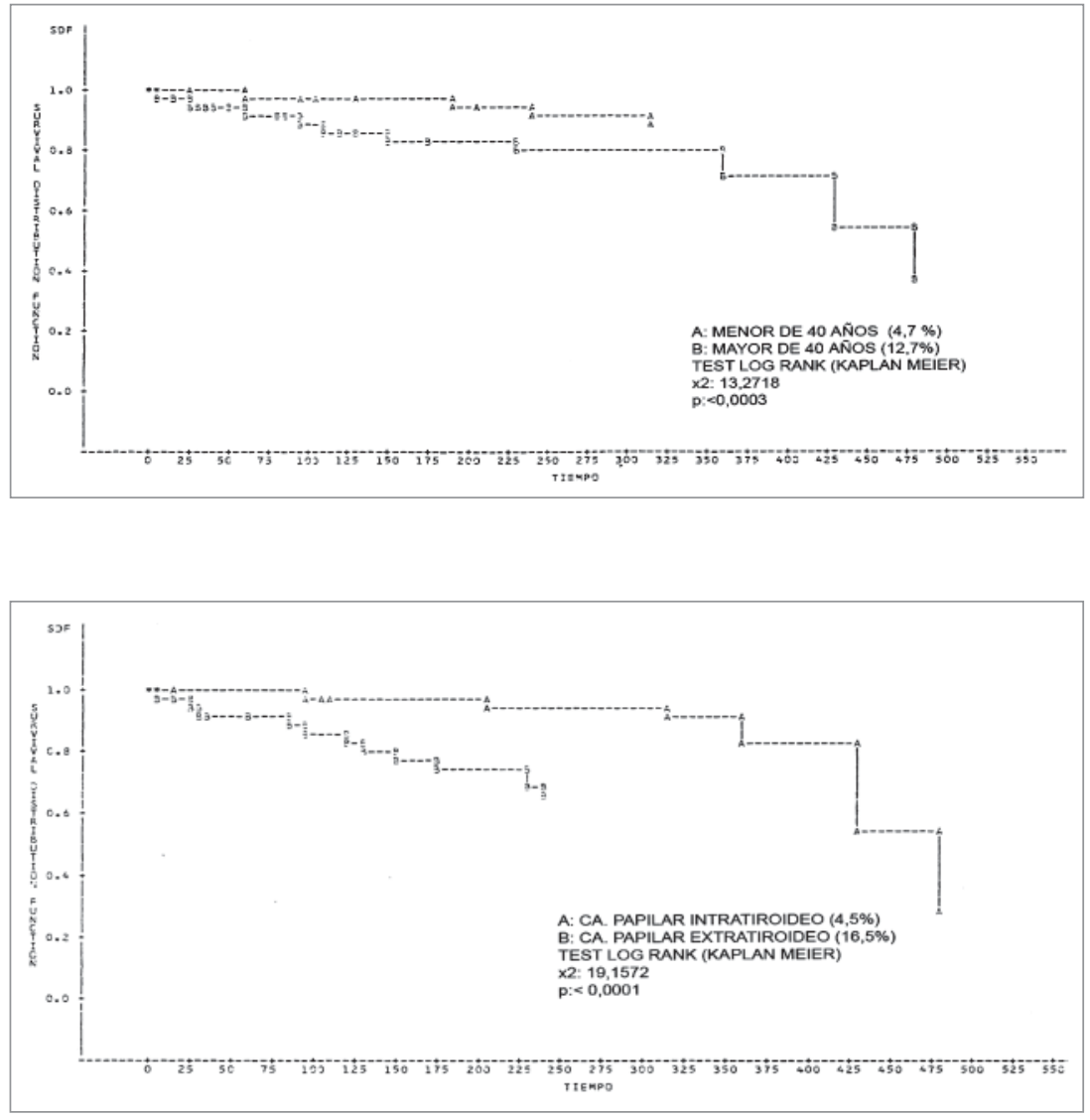

Figura 1. Curva de supervivencia del cáncer diferenciado de tiroides según edad al momento del diagnóstico.
Figura 2. Curva de supervivencia del cáncer papilar según extensión anatómica 

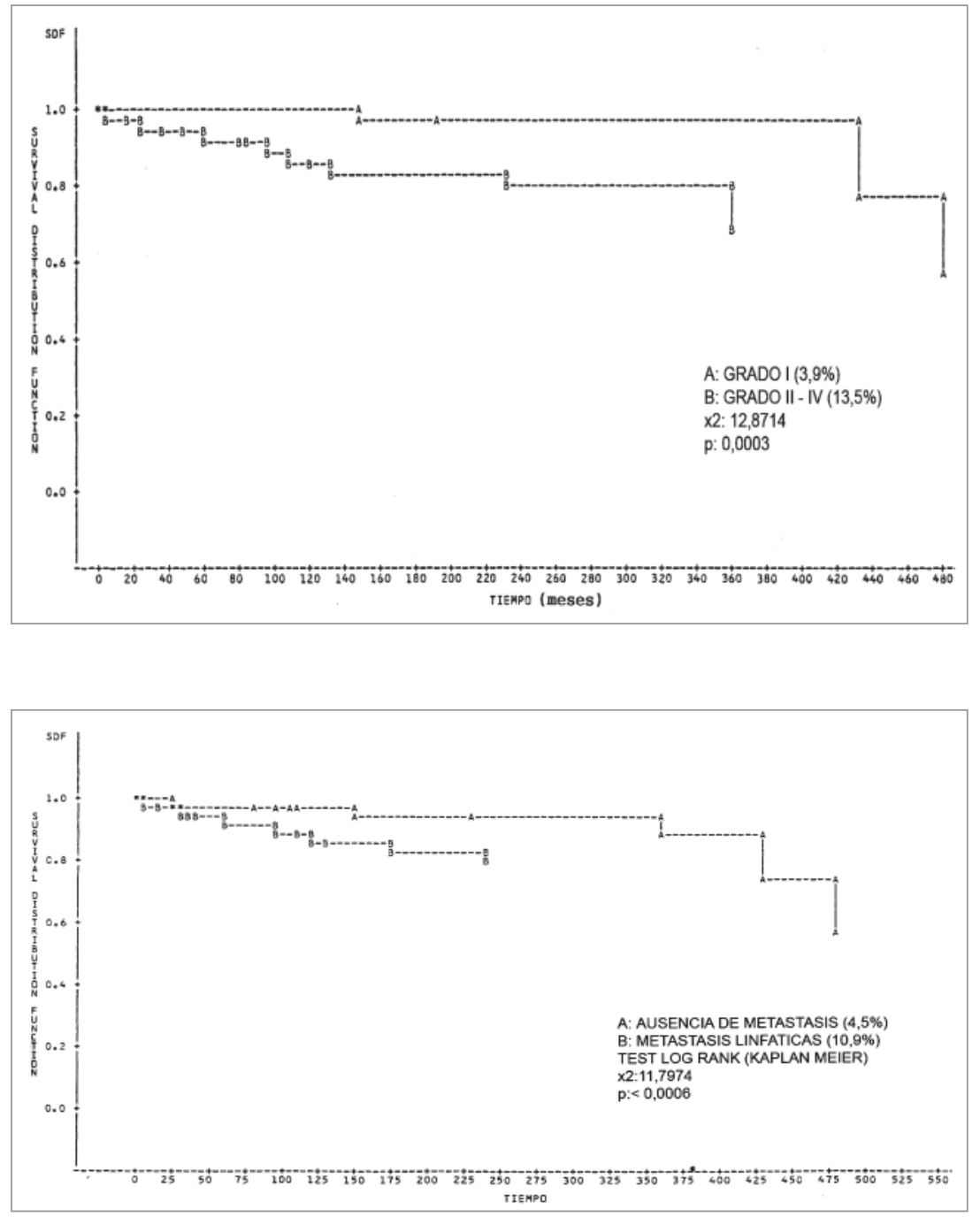

Figura 3. Curva de supervivencia del cáncer diferenciado de tiroides según grado histológico.
Figura 4. Curva de supervivencia del cáncer diferenciado de tiroides según existencia de metástasis linfáticas al momento del diagnóstico.
Al estudiar las variables predictoras de supervivencia en 296 casos de carcinoma papilar según el modelo de Cox, resultaron significativas las metástasis a distancia $(\mathrm{p}<0,002)$, extensión anatómica $(p<0,006)$ y la edad $>$ de 40 años $(p<0,008)$; en 123 carcinomas foliculares sólo las metástasis a distancia $(\mathrm{p}<0,0002)$ fueron significativas.

\section{Discusión}

La serie presentada comprende 477 pacientes tratados primariamente en el Hospital San Juan de Dios entre 1948 y 1992. Nos llamó la atención la alta frecuencia de cáncer folicular, 30,6\%. Como consecuencia de la yodación de la sal por Ley de la República, a contar desde 1978 se ha producido un cambio significativo en la proporción de las frecuen- cias del cáncer papilar versus folicular (papilar: de $65 \%$ a $86,5 \%$ y folicular $35 \%$ a $13,5 \%)^{20,22}$. Por otra parte, se ha observado un cambio en la proporción relativa de las sub categorías patológicas en ambos tipos histológicos. También cabe destacar el hallazgo de cáncer tiroideo en bocio multinodular (45,5\%).

Varios autores han estudiado la supervivencia de los pacientes operados por cáncer diferenciado de tiroides, entre ellos Woolner y cols $^{5}$, quienes precisaron hace más de 30 años, el concepto de los factores del pronóstico y que fueron destacados posteriormente en otras publicaciones de la Clínica Mayo. También, han aportado importantes contribuciones al tema, los investigadores de la Universidad de Chicago 9 , la Clínica Lahey ${ }^{10,11}$, la Universidad de Houston, Texas ${ }^{12}$, Memorial Sloan-Kettering Cancer Center, New York ${ }^{13}$, el Estudio Cooperativo 
Nacional del Tratamiento del Cáncer de Tiroides del NTCTCS $^{14}$ y en Europa el grupo de la EORTC ${ }^{15-18}$.

El análisis univariable de toda la serie demostró que la edad del paciente en el momento del diagnóstico, constituyó un factor pronosticador muy significativo ( $p<0,0003)$; sin embargo, el modelo de Cox, demostró que esta variable fue significativa sólo en el cáncer papilar $(\mathrm{p}<0,0089)$ porque el número reducido de pacientes con cáncer folicular invasor no permitió aplicar dicha metódica.

Al comparar la supervivencia por género no hubo una diferencia estadísticamente significativa $(\mathrm{p}>0,0562)$. En la literatura extranjera se observa una correlación directa entre el grado histológico y la mortalidad por el cáncer. La curva de supervivencia de esta serie mediante el análisis univariable, demostró una diferencia significativa favorable para el grado I ( $<<0,0003$ ) (Figura 4), sin embargo, el modelo de Cox encontró un valor escasamente significativo ( $\mathrm{p}>0,0594)$, ya que el número de pacientes con cáncer folicular, fue menor que el exigido por el método.

No hubo diferencias significativas en la tasa de supervivencia al comparar el cáncer papilar con el folicular $(p>0,2611)$; resultados semejantes a lo publicado por la Clínica Mayo ${ }^{7,8}$ y otros ${ }^{15,17}$.

Al comparar la mortalidad por extensión anatómica, en el cáncer papilar, esta fue 25 veces superior en la forma extratiroídea que en la intratiroídea $(\mathrm{p}<0,0001)$ y en la forma invasora del cáncer folicular fue 20 veces mayor que la mínimamente invasora $(\mathrm{p}<0,0001)$.

El método de Cox demostró una correlación positiva entre el tamaño del tumor y la supervivencia en el cáncer papilar.

La frecuencia de las metástasis linfáticas iniciales en esta serie, fue comparable con la de otros ${ }^{10}$. Diversas comunicaciones señalan que la presencia de metástasis linfáticas iniciales y especialmente las distantes, tienen un efecto desfavorable para el pronóstico ${ }^{6,8,10,12,13}$; el modelo de Cox, demostró una diferencia significativa en la supervivencia respecto a la presencia o no de metástasis a distancia iniciales en ambos tipos histológicos $(\mathrm{p}<0,0001)$.

Diversos investigadores han utilizado sistemas de puntaje para definir los grupos de bajo y alto riesgo de recidiva y muerte por la enfermedad sobre la base de factores pronosticadores ${ }^{23,24}$ :

TNM (Tumor, Nodes (lymph nodes), Metastasis). American Joint Committee on Cancer.

AGES (Age, Grade, Extension, Size) Clínica Mayo.

MACIS (Metastasis, Age, Completeness of surgery, Invasion, Size) Clínica Mayo.

AMES (Age, Metástasis, Extension, Size) Clínica Lahey.
DAMES (DNA, Age, Metastasis, Extension) Clínica Lahey.

EORTC (Edad, Género, Extensión, Metástasis en un sitio, Metástasis en más de un sitio).

La aplicabilidad del sistema TNM ha sido cuestionada por algunos autores en el extranjero. Un informe del NCDB (National Cancer Data Base) en un análisis de 53.856 casos de carcinoma diferenciado validó el sistema AMES sólo para el cáncer folicular y concluyó que para que el sistema TNM fuera operativo debería simplificarse ${ }^{21}$.

El NTCTCS (National Thyroid Cancer Treatment Cooperative Study) desarrolló un sistema TNM sobre la base de las siguientes variables: edad del paciente, tamaño e histología del tumor, multifocalidad, invasión extra glandular, metástasis y diferenciación celular ${ }^{14}$. Sin embargo, los resultados fueron analizados después de un promedio de seguimiento de 3 años 4 meses, período muy breve para evaluar estos factores.

\section{Conclusión}

Analizando los resultados según el método de Regresión Múltiple o Análisis de Riesgos Proporcionales de Cox, se puede concluir que los factores que determinan que el pronóstico del cáncer diferenciado del tiroides sea desfavorable en esta serie son: las metástasis a distancia iniciales, la extensión anatómica avanzada, la edad $>$ de 40 años en el momento del diagnóstico y el tamaño del tumor $>$ de 10 $\mathrm{mm}$ para el cáncer papilar; la extensión anatómica y las metástasis a distancia iniciales para el cáncer folicular.

No es posible efectuar estudios prospectivos y randomizados sobre la evolución del cáncer diferenciado de tiroides, ya que sería necesario analizar un gran número de pacientes durante un período muy prolongado de observación.

\section{Agradecimientos:}

Expresamos nuestros más profundos agradecimientos a la Dra. Violeta Díaz T., Profesora Asociada de Medicina, Directora del Departamento de Neurología y de Neurocirugía del Hospital Clínico de la Universidad de Chile, por su valioso apoyo en el diseño y análisis estadístico de este manuscrito.

\section{Referencias}

1. Parker SL, Tong T, Bolden S, Wingo PA. Cancer statistics, 1997. CA Cancer J Clin. 1997; 47:5-27.

2. Schlumberger MJ, Filetti S, Hay ID. Nontoxic goiter and thyroid neoplasia. En William Textbook of Endocri- 
nology; $10^{\circ}$ Edition. Editorial WD Saunders Company 2003. Capítulo 13: pág. 483-99.

3. López E, Torres J, Domínguez M, Volpato R, Martínez V, Maira J, y cols. Cáncer del Tiroides: Aspectos clínicos de 320 casos recolectados en 27 años. Rev Med Chile 1982;110:123-32.

4. Woolner LB, Beahrs OH, Black BM, Mcconahey WM, Keating Fr Jr. Classification and prognosis of thyroid carcinoma. A study of 885 cases observed in a thirty year period. Am J Surg. 1961;102:354-87.

5. Woolner LB. Thyroid carcinoma: pathologic classification with data on prognosis. Semin Nucl Med. 1971;1:481-502.

6. McConahey WM, Hay ID, Woolner LB, van Heerden JA, Taylor WF. Papillary thyroid cancer treated at the Mayo Clinic, 1946 through 1970: initial manifestations, pathologic findings, therapy, and outcome. Mayo Clin Proc. 1986;61:978-96.

7. Hay ID. Pronostic factors in Thyroid Carcinoma. Thyroid Today 1989;12:1-9.

8. Hay ID, Thompson GB, Grant CS, Bergstralh EJ, Dvorak $\mathrm{CE}$, Gorman CA, et al. Papillary thyroid carcinoma managed at the Mayo Clinic during six decades (19401999): temporal trends in initial therapy and long-term outcome in 2444 consecutively treated patients. World J Surg. 2002;26:879-85

9. DeGroot LJ, Kaplan EL, McCormick M, Straus FH. Natural history, treatment, and course of papillary thyroid carcinoma. J Clin Endocrinol Metab. 1990;71:414-24.

10. Cady B, Rossi R. An expanded view of risk-group definition in differentiated thyroid carcinoma. Surgery 1988;104:947-53.

11. Rossi RL, Nieroda C, Cady B, Wool MS. Malignancies of the thyroid gland: The Lahey Clinic experience. Surg Clin North Am. 1985;65:211-30.

12. Samaan NA, Schultz PN, Hickey RC, Goepfert H, Haynie TP, Johnston DA. The results of various modalities of treatment of well differentiated thyroid carcinomas: a retrospective review of 1599 patients. J Clin Endocrinol Metab. 1992;75:714-20.

13. Shah JP, Loree TR, Dharker D, Strong EW, Begg C, Vlamis V. Prognostic factors in differentiated carcinoma of the thyroid gland. Am J Surg. 1992;164:658-61.
14. Sherman SI, Brierley JD, Sperling M, Ain KB, Bigos ST, Cooper DS, et al. Prospective multicenter study of thyroids carcinoma treatment: initial analysis of staging and outcome. National Thyroid Cancer Treatment Cooperative Study Registry Group. Cancer 1998;83:101221.

15. Byar DP, Green SB, Dor P, Williams ED, Colon J, van Gilse HA, et al. A prognostic index for thyroid carcinoma. A study of the E.O.R.T.C. Thyroid Cancer Cooperative Group”. Eur J Cancer 1979;15:1033-41.

16. Tubiana M, Schlumberger MJ, Rougier P, Laplanche A, Benhamou E, Gardet P. Long-term results and prognostic factors in patients with differentiated thyroid carcinoma. Cancer 1985; 15;55:794-804.

17. Schlumberger MJ. Papillary and follicular thyroid carcinoma. N Engl J Med. 1998;338:297-306.

18. Tennvall J, Biörklund A, Möller T, Ranstam J, Akerman $\mathrm{M}$. Is the EORTC prognostic index of thyroid cancer valid in differentiated thyroid carcinoma? Retrospective multivariate analysis of differentiated thyroid carcinoma with long follow-up. Cancer 1986;57:1405-14.

19. Cox DR. Regression models and life table (with discussion). J R Stat Soc. 1972;34:187-220.

20. Tapia L, Pereira A, Cisternas D, Carmi A, Plaza C, Rodríguez $\mathrm{P}$, y cols. Relación entre la frecuencia del cáncer folicular y papilar antes y después de la yodación de la sal. Bol S J de Dios 2001;48:1-5.

21. Hundahl SA, Fleming ID, Fremgen AM, Menck HR. A National Cancer Data Base report on 53.856 cases of thyroid carcinoma treated in the U.S. 1985-1995". Cancer 1998;83:2638-48.

22. González M, Andrades P, Contreras O, Irarrázabal V, Paladines P, Inturias R, y cols. Sobrevida en cáncer de tiroides: seguimiento de 365 pacientes por un período de 37 años. Rev Chil Cir. 2002;54:329-35.

23. Iribarren $\mathrm{O}$, Velasco $\mathrm{N}$, Huidobro $\mathrm{P}$, Núñez $\mathrm{H}$, Villalón M, Madariaga J, y cols. Evolución y factores de pronóstico en cáncer diferenciado de tiroides. Rev Chil Cir. 2009;61:136-41.

24. Iribarren $\mathrm{O}$, Madariaga $\mathrm{J}$, Zaffiri $\mathrm{M}$, Huidobro $\mathrm{P}$, Mertens M, Iribarren G. Factores pronóstico de las variantes de cáncer papilar de tiroides. Rev Chil Cir. 2010;62:228-33. 